\title{
10q23/PTEN Locus Deletion Negative
}

National Cancer Institute

\section{Source}

National Cancer Institute. 10q23/PTEN Locus Deletion Negative. NCI Thesaurus. Code C160306.

A genetic finding indicating that deletion of 10q23/PTEN has not been detected in a sample. 